\title{
Statistical Monitoring of Condemnations from Slaughterhouses
}

\author{
Flavie Vial*1, Sarah Thommen² and Leonhard Held ${ }^{2}$ \\ 'Vetsuisse Faculty, Veterinary Public Health Institute, Bern, Switzerland; '2Institute of Social and Preventive Medicine, Zürich, \\ Switzerland
}

\section{Objective}

We evaluate the performance of the improved Farrington algorithm for the detection of simulated outbreaks in meat inspection data.

\section{Introduction}

Meat inspection data are routinely collected over several years providing the possibility to use historical data for constructing a baseline model defining the expected normal behaviour of the indicator monitored. In countries in which the reporting of data is compulsory (e.g. in the EU), coverage of the majority of the slaughtered population is ensured.

\section{Methods}

This study focuses on the whole carcass condemnations (WCC) of cattle slaughtered in Switzerland between 2007 and 2012 (1). The monthly time series was retrospectively modelled using the function $\{\mathrm{hhh} 4$ \} in the $\mathrm{R}$ \{surveillance\} package (2). The mean monthly incidence was decomposed additively into an auto-regressive component; and an endemic component multiplied by the offset to adjust for variation in the number of total animals slaughtered per month. The retrospective model was used to simulate a baseline series to which an outbreak was added at a random starting point $\mathrm{t}_{\mathrm{i}}$. Outbreak sizes were randomly generated as Poisson with mean equal to $\mathrm{k}$ times (with $\mathrm{k}$ from 2 to 10) the standard deviation of the baseline count at $\mathrm{t}_{\mathrm{i}}$ (1000 simulations for each $\mathrm{k}$ ). The improved Farrington algorithm (3) was applied for outbreak detection using the \{faringtonFlexible function:

\section{Results}

Algorithm A includes parameters chosen according to the results of the retrospective analysis (no trend and no seasonality). Algorithm $B$ includes more historic data $(b=3$ instead of $b=2)$ and considers monthly seasonal effects $(\mathrm{w}=0)$. Both algorithms produced less than one false alarm per year. In terms of probability of detection (POD), algorithm A outperforms algorithm B at the detection of larger outbreaks ( $\mathrm{k}=5$ to 10 ), but the latter is more appropriate when looking to detect smaller outbreaks (Figure 1).

\section{Conclusions}

The improved Farrington algorithm led to low false positive rate (FPR) but the POD was low for small outbreaks. Furthermore, more than $50 \%$ of the outbreak cases had already occurred by the time the outbreak was detected. The low temporal resolution of this dataset currently limits its use for early detection.

$$
\begin{gathered}
E\left[y_{t}\right]=\mu_{t}=\left(\alpha+\beta t+f_{t}\right) * e_{t} \\
\operatorname{Var}\left(y_{t}\right)=\theta * \mu_{t} \text { with } \theta>1
\end{gathered}
$$

Table 1: Outbreaks attributes in WCC data (averaged over 1000 simulations for each $\mathrm{k}$ )

\begin{tabular}{|c|c|c|c|}
\hline $\mathrm{k}$ & Duration (month) & Final size & Extra WCC/month \\
\hline 2 & 3.9 & 33.1 & 8.5 \\
\hline 3 & 4.2 & 49.6 & 11.8 \\
\hline 4 & 4.3 & 66.5 & 15.5 \\
\hline 5 & 4.4 & 83.5 & 19.0 \\
\hline 6 & 4.6 & 99.6 & 21.7 \\
\hline 7 & 4.7 & 116.2 & 24.7 \\
\hline 8 & 4.7 & 132.6 & 28.2 \\
\hline 9 & 4.9 & 149.6 & 30.5 \\
\hline 10 & 4.9 & 166.3 & 33.9 \\
\hline
\end{tabular}

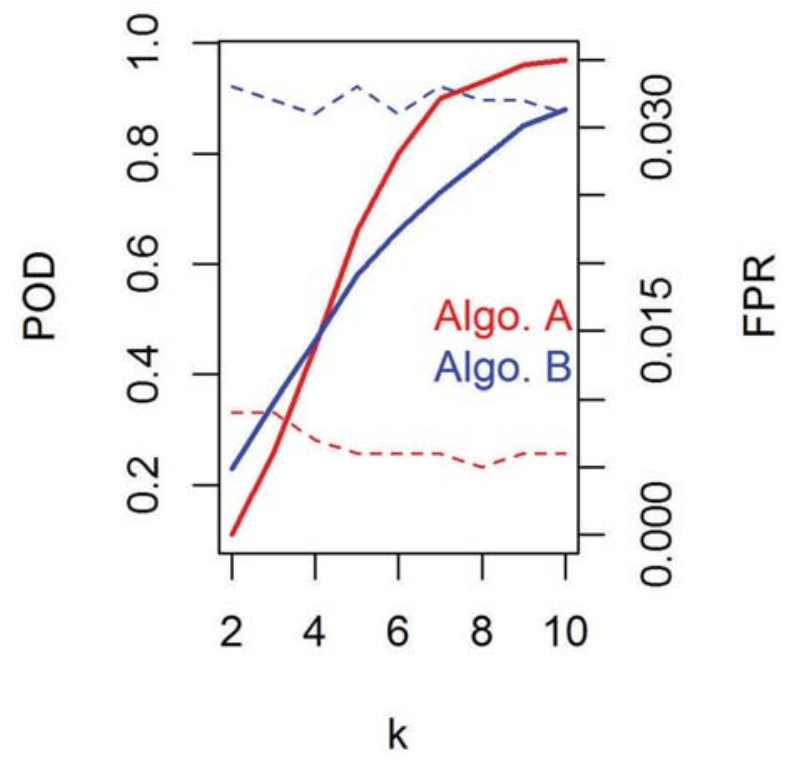

POD and FPR of algorithms A \&B for different outbreak sizes (k)

Keywords

Meat inspection; veterinary public health; syndromic surveillance

\section{Acknowledgments}

Marion Zumbrunnen extracted the data \& Sebastian Meyer helped with the $\mathrm{R}$ programming.

\section{References}

1.Vial F, Reist M. Evaluation of Swiss slaughterhouse data for integration in a syndromic surveillance system. BMC Vet Res. 2014 Jan;10:33.

2.Höhle M. \{ surveillance\} : An R package for the monitoring of infectious diseases. Comput Stat. 2007 Aug;22(4):571-82.

3.Noufaily A, Enki DG, Farrington CP, Garthwaite PH, Andrews NJ, Charlett A. An improved algorithm for outbreak detection in multiple surveillance systems. Stat Med. 2013 Mar 30;32(7):1206-22.

\section{*Flavie Vial}

E-mail: flavie.vial@vetsuisse.unibe.ch 